\title{
Effect of the Use of Intranasal Spray of Essential Oils in Patients with Perennial Allergic Rhinitis: A Prospective Study
}

\author{
Davide Caimmi ${ }^{a}, b$ Catherine Neukirch ${ }^{c}$ Renaud Louis $^{d}$ Olivier Malard $^{e}$ \\ Gabriel Thabut ${ }^{c}$ Pascal Demoly a, b \\ aDepartment of Pulmonology, Division of Allergy, Hôpital Arnaud de Villeneuve, University Hospital of Montpellier, \\ University of Montpellier, Montpellier, France; bUMR-S 1136 INSERM-Sorbonne Université, Equipe EPAR - IPLESP, \\ Paris, France; 'Departments of Pneumology A and B and INSERM U1152, Bichat-Claude Bernard University Hospital, \\ Paris, France; ${ }^{d}$ Department of Pulmonary Medicine, CHU Sart-Tilman, IGIGA Research Group, University of Liege, \\ Liège, Belgium; 'Service d'ORL et de Chirurgie Cervico-Faciale, University Hospital of Nantes, Hôtel Dieu, Nantes, \\ France
}

\section{Keywords}

Allergic rhinitis · Essential oils · Management · Control

\begin{abstract}
Introduction: Among allergic rhinitis (AR) symptoms, nasal obstruction particularly affects the quality of life. Antihistamines and intranasal corticosteroids are the most frequently prescribed symptomatic drugs, but their efficacy is often incomplete. Essential oils (EO) have shown an anti-inflammatory effect and potential in treating patients with AR. The aim of this study was to evaluate the effectiveness of a hypertonic EO-based nasal spray on perennial AR (PAR) symptoms. Methods: This prospective, open-label, non-randomized, multicentric trial included 43 patients with PAR sensitized to mites, not controlled for more than a year. All were treated with Puressentiel ${ }^{\circledR}$ Respiratory-Decongestant Nasal Spray for 30 days. Their usual treatment remained unchanged during the study period. Before and after treatment, each participant filled out a rhinitis questionnaire, the Allergic Rhinitis Control Test (ARCT). A nasal inspiratory peak flow (NIPF) was performed. Results: The mean ARCT was 16.4 and 20.5
\end{abstract}

at D0 and D30, respectively ( $p<0.001)$; the mean increase between D0 and D30 was 4.1 ( $p<0.001)$. The proportion of patients with controlled rhinitis after 30 days of treatment was 69.8 versus $14 \%$ before treatment $(p<0.001)$. The mean NIPF was $86.5 \mathrm{~L} / \mathrm{min}$ and $105.1 \mathrm{~L} / \mathrm{min}$ at D0 and D30, respectively ( $p<0.001$ ); the mean increase between D0 and D30 was 18.5 L/min. Conclusion: A hypertonic EO-based nasal spray could be a new and natural option in the management of PAR. It could also be used as an add-on therapy when nasal symptoms are not fully controlled.

(c) 2020 The Author(s)

Published by S. Karger AG, Basel

\section{Introduction}

Allergic rhinitis (AR) is one of the most common manifestations of immunoglobulin E-mediated inflammation after exposure of the nasal mucosa to allergens. The classical symptoms of $\mathrm{AR}$ are nasal congestion, nasal itch, rhinorrhea, and sneezing [1]. AR is very common in

Edited by H.-U. Simon, Bern.
C) 2020 The Author(s)

Published by S. Karger AG, Basel

This is an Open Access article licensed under the Creative Commons Attribution-NonCommercial-4.0 International License (CC BY-NC) (http://www.karger.com/Services/OpenAccessLicense), applicable to the online version of the article only. Usage and distribution for commercial purposes requires written permission.
Davide Caimm

Department of Pulmonology, Division of Allergy

Hôpital Arnaud de Villeneuve, CHU de Montpellier

371, Ave. du Doyen Gaston Giraud, FR-34090 Montpellier (France) davide.caimmi@gmail.com 
Western countries, affecting $20-30 \%$ of the population, and evidence suggests that the prevalence of the disorder is increasing [2].

AR impairs quality of life, sleep, and social activities [3]. It is a significant cause of reduced productivity at work and absence from school. Poor sleep quality may induce diurnal somnolence. The impact is correlated with the severity of symptoms [1].

The main challenges in AR relate to its treatment. Overall, the guidelines for treatment are based on the frequency, duration, and severity of symptoms [4]; the treatment goal being the relief of symptoms and a reduction of their impact on quality of life. Therapeutic options available to achieve this goal include avoidance measures, oral antihistamines, intranasal corticosteroids, antileukotrienes, nasal vasoconstrictors, and allergen immunotherapy [1]. The guidelines (ARIA) recommend different pharmacological treatments, which can be increased step by step according to disease severity [4]. Antihistamines and intranasal corticosteroids are the most frequently prescribed drugs for relieving AR symptoms [5] and are recommended as the first-line therapy, either alone or in combination. However, the effects of antihistamines on symptoms, especially nasal congestion, are modest [6]. Studies and meta-analyses have shown that intranasal corticosteroids are superior to antihistamines and leukotriene receptor in controlling the symptoms, particularly nasal congestion, in patients with moderate-to-severe AR $[7,8]$. However, to achieve maximum benefit and relief, nasal corticosteroids should be used continually for 3-4 weeks, thus underlining the importance of patient adherence [9]. Indeed, adherence is imperative in the management of AR, and lack of adherence can be an obstacle to effective treatment. One reason for non-adherence is that, although intranasal corticosteroids are the most effective pharmacotherapy for AR, their overall efficacy is moderate $[6,10]$, and most patients are concerned about long-term steroid treatment. Another common reason is the bothersome side effects, including nasal dryness, burning, stinging, sneezing [9], and bleeding [11].

There has been a recent increase in interest in the general population regarding complementary and alternative medicine, and several review articles have been published on their role in treating allergic disease, including $A R$ [12-14]. Instead of rejecting them bluntly, nondrug interventions for which beliefs are strong and the current level of evidence of their interest or deleterious effects is low need to be considered. Aromatherapy, especially the direct inhalation of aroma compounds of essential oil (EO) fragrance, has long been used for various inflammatory diseases [15]. Recently, EOs containing monoterpenes have shown an anti-inflammatory effect in a murine model of asthma [16], as well as potential in treating patients with AR [17].

However, despite the potential of EOs in the treatment of AR, there have been few clinical trials on human AR patients that directly measure the effects on symptoms. Additional studies are needed to fully evaluate the effectiveness and potential long-term benefits of these compounds. The aim of the present study was to evaluate the efficacy on symptoms, particularly nasal congestion, of a nasal spray containing $4 \mathrm{EOs}$ in patients with perennial AR (PAR).

\section{Materials and Methods}

\section{Patients}

Forty-three patients (33 women and 10 men) were recruited during a follow-up visit to the outpatient allergy clinic of 3 academic hospitals: 2 located in France (18 and 12 patients) and 1 in Belgium (13 patients). All patients fulfilled the following inclusion criteria: (i) over 18 years of age, (ii) diagnosed with AR according to ARIA and presenting clinical symptoms, (iii) over 1-year history of PAR, and (iv) with positive skin test results (wheal $\geq 3 \mathrm{~mm}$ compared with control) for at least 1 perennial allergen. Exclusion criteria were as follows: patients (i) receiving allergen immunotherapy, (ii) having had aromatherapy experience in the past, (iii) having had upper respiratory infection within the 8 previous weeks before inclusion or with chronic rhinosinusitis, (iv) with a history of drug allergy, (v) who were pregnant or breastfeeding, and (vi) with a history of neurological or psychiatric diseases.

\section{Ethics, Consent, and Permissions}

The study was approved by a local French Ethics Committee (Registration No. ID-RCB: 2018-A00836-49) and registered on ClinicalTrials.gov (No. NCT03963076). All participants signed an informed consent document, including consent for publication.

\section{Study Protocol}

This was a multicentric, prospective open-label, non-randomized study. All patients used Puressentiel ${ }^{\circledR}$ Respiratory-Decongestant Nasal Spray (PRDNS) (Puressentiel Laboratory, Brussels, Belgium), which is a combination of hypertonic seawater and organic rosemary floral water with 4 EOs (ravintsara, geranium, eucalyptus radiata, and niaouli). All ingredients contained in the spray are natural and comply with the BBDEO quality charter (biochemically and botanically defined EOs). The spray contains no propellant gas.

The patients were asked to use PRDNS twice a day for 4 weeks ( 2 sprays in each nostril morning and evening, every day at 8 a.m. and 8 p.m.). Evaluation procedures (listed below) at baseline before PRDNS administration (D0) were conducted at 10 a.m. The procedures after 4 weeks of PRDNS administration (D30) were also conducted at 10 a.m. Participants were not allowed to take any anti-allergic treatment (systemic or topical) apart from their usual one, which they continued without modification throughout the 
Table 1. Changes in Allergic Rhinitis Control Test and NIPF between baseline (D0) and after 30 days of treatment (D30)

\begin{tabular}{lcccc}
\hline Parameter & $N$ & D0 & D30 & $p$ value \\
\hline Controlled rhinitis (score $>19), n(\%)$ & 43 & $6(14.0 \%)$ & $31(69.8 \%)$ & $<0.001^{\mathrm{a}}$ \\
ARCT, mean (SD) & 43 & $16.4(3.2)$ & $20.5(3.7)$ & $<0.001^{\mathrm{b}}$ \\
NIPF (L/min), mean (SD) & 42 & $86.5(37.3)$ & $105.1(32.7)$ & $<0.001^{\mathrm{b}}$ \\
\hline
\end{tabular}

ARCT, Allergic Rhinitis Control Test; NIPF, nasal inspiratory peak flow. ${ }^{\text {a } M c N e m a r ~ t e s t . ~}{ }^{b}$ Student's $t$ test for paired data.

study (40 patients). The 3 patients who had no treatment before inclusion in the study were not allowed to take any medication.

A spray bottle of PRDNS $(15 \mathrm{~mL})$ containing 4 EOs was provided to each patient. After 4 -week administration, the patient brought back the bottle, which was weighed to assess compliance. All data are available at the study sites.

\section{Outcomes}

Rhinitis Control and Symptoms

AR control was assessed using the Allergic Rhinitis Control Test (ARCT), a self-completed questionnaire [18] consisting of 5 questions, scored from 1 to 5 . These individual scores were then added up to obtain a score ranging from 5 (worst score) to 25 (best score). An ARCT $\geq 20$ was regarded as controlled AR; a lower score indicated uncontrolled AR [12]. The ARCT score was considered the primary outcome. It was measured at baseline (D0) and 4 weeks after PRDNS administration (D30). Each subject also filled out a daily diary and was asked 5 questions concerning perception of discomfort and side effects (nasal dryness, burning, stinging, sneezing, and bleeding).

Nasal Inspiratory Peak Flow

Nasal inspiratory peak flow (NIPF), which measures basal flow and gives a direct measure of nasal obstruction (NO), was considered the secondary outcome [19]. The technique consists of application of a face mask over the nose and obtaining 3 satisfactory maximal inspirations with the mouth closed. The highest result of the 3 was taken as the NIPF [19]. Measurements of NIPF were performed at baseline (D0) before PRDNS administration and 4 weeks later (D30).

\section{Statistical Analysis}

Stata software (StataCorp LLC, College Station, TX, USA) version 15 and R software version 3.3 (the R Foundation for Statistical Computing, Vienna, Austria) were used for data management and data analysis, respectively. Continuous variables were summarized with descriptive statistics (number, mean, SD, range, and median), and frequency counts and percentages within each category were provided for categorical data. Statistics were computed for patients with available (i.e., non-missing) data. No imputation was performed. Comparison of patient characteristics before and after treatment was done using Student's $t$ test for data in the case of continuous variable and the $\mathrm{McNemar}$ test for categorical variable. Differences between groups were considered statistically significant if $p$ values were $<0.05$. No attempt was made to adjust for multiple comparisons.
Sample Size Determination

This study was designed to detect an absolute improvement of $20 \%$ between D0 and D30 in the score of nasal symptoms, assuming that all patients at baseline were not controlled. To detect such a difference with $90 \%$ power ( $\alpha=0.05,2$-tailed test), 60 patients are required, assuming no dropout during the study. Due to enrollment issues, we decided to stop enrollment after 43 patients had been included.

\section{Results}

\section{Patients}

All patients were between 22 and 60 years of age, mean 36.7 years (SD: 11$)$. They were classified as persistent mild $(n=6)$ or persistent moderate-to-severe PAR $(n=37)$, according to the ARIA guidelines [4]. All were symptomatic at inclusion, with persistent NO. Among the 43 patients, all had a positive prick reaction to house dust mites, 8 to house dust mites alone, 8 to house dust mites and cat dander, 16 to house dust mites and a seasonal allergen (grass, tree, or weed pollens). Forty patients were treated on a regular basis with antihistamines, either alone (19 patients) or in combination with intranasal steroids (21 patients), and in addition, some occasionally used decongestants ( 5 patients) or oral corticosteroids (2 patients). Three patients had no regular treatment but used decongestants as needed. All patients were nonsmokers. Patients were included between March and June 2019. All 43 patients completed the 30 days of treatment.

\section{Allergic Rhinitis Control Test}

ARCT was assessable in the 43 patients at D0 and D30. The mean ARCT (Table 1) was 16.4 (3.2; range: 10-22) and 20.5 (3.7) at D0 and D30, respectively $(p<0.001)$, and the mean increase between D0 and D30 was 4.1 (3.7), $p<$ 0.001 (Table 1 ). The proportion of patients with controlled rhinitis after 30 days of treatment with intranasal PRDNS administration was 69.8 versus $14 \%$ before treat- 
Fig. 1. Individual and median (red triangles) values of Allergic Rhinitis Control Test (ARCT) before (D0) and after 1 month of treatment (D30) with Puressentiel ${ }^{\circledR} \mathrm{Re}$ spiratory-Decongestant Nasal Spray (PRDNS).

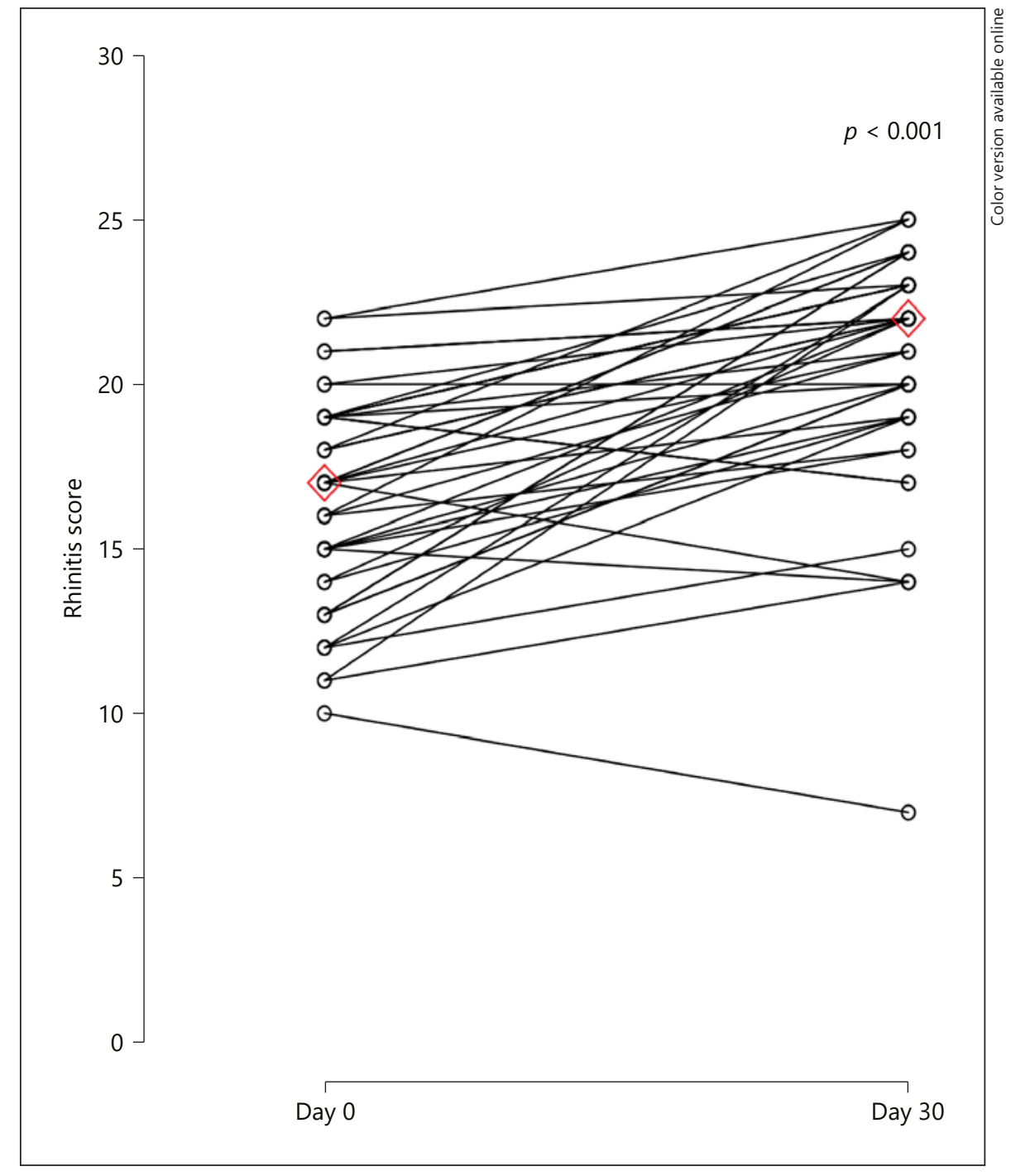

ment ( $p<0.001$, McNemar test for paired data). Six patients had controlled rhinitis at D0 and remained controlled at D30. If we consider only the patients with uncontrolled rhinitis at D0 $(n=37)$, the increase in ARCT between D0 and D30 remains highly significant $(p<$ 0.001 ). Individual values before and after treatment are shown in Figure 1.

\section{Nasal Inspiratory Peak Flow}

NIPF was assessable in 42 patients at D0 and D30. The mean NIPF (Table 1) was $86.5 \mathrm{~L} / \mathrm{min}$ (37.3; range: 30 $200)$ and $105.1 \mathrm{~L} / \mathrm{min}(32.7)$ at D0 and D30, respectively $(p<0.001)$, and the mean increase between D0 and D30 was $18.5(31.0) \mathrm{L} / \mathrm{min}$ (Table 1). Individual values before and after treatment are shown in Figure 2.

\section{Adverse Side Effects}

No adverse events were declared.

\section{Compliance}

To assess compliance, the weight of the PRDNS bottles was measured prior to the first use and at the end of the study. The mean baseline weight was $109.4 \mathrm{~g}$ (DS: 0), and post-study weight $70.2 \mathrm{~g}$ (DS: 12.6). The average amount of PRDNS used was $39.2 \mathrm{~g}$ (20.8), with variations from 11.1 to $133.9 \mathrm{~g}$. These values showed that patients did not always respect the prescribed dose. A sub-analysis of the efficacy of the product based on different doses did not show significant results, likely due to the low number of patients. 
Fig. 2. Individual and median (red triangles) values of nasal inspiratory peak flow (NIPF) before (D0) and after 1 month of treatment (D30) with Puressentiel ${ }^{\circledR}$ Respiratory-Decongestant Nasal Spray (PRDNS).

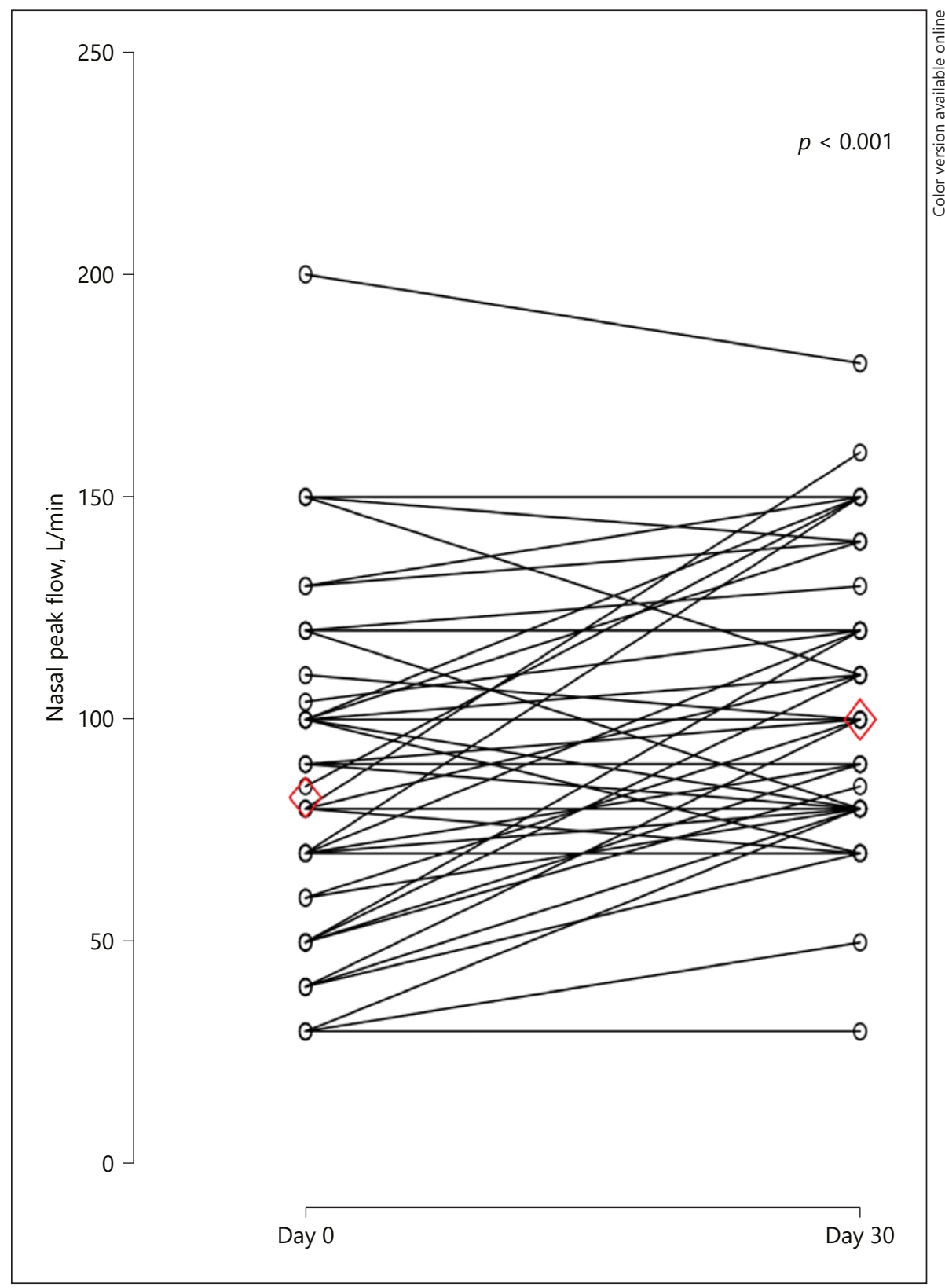

\section{Discussion/Conclusion}

The current study was designed to investigate the effects of a nasal spray (PRDNS) of EOs on the perception of PAR symptoms using a validated self-completion questionnaire (ARCT) and nasal patency using a direct and objective measure of NO (NIPF). A marked improvement in these 2 outcomes was observed after 4 weeks of PRDNS exposure (Table 1; Figs. 1, 2).
Disease control is now being considered as a major goal in the management of patients with $\mathrm{AR}$, as already mentioned in the ARIA guidelines [4]. In this report, we used the ARCT, a reproducible, quick, and easy test to perform [18]. The latter is a 5-item self-assessment questionnaire [18], which had been validated by testing in 902 patients before treatment and 2 weeks after treatment. The score at inclusion correlated significantly with the patient's overall clinical status and the impact of AR on social and sports activities, and an increase in the score 
was observed after 2 weeks of treatment [18]. A score of 20 was found to be the optimal cutoff for poor versus wellcontrolled rhinitis [18]. In our study, a significant increase in ARCT score was noted (Table 1; Fig. 1), 70\% of the patients reported good control (ARCT $\geq 20$ ) at D30 versus $14 \%$ before PRDNS administration. The mean improvement of ARCT was 4.1, which is above the minimum clinical important difference (data not shown). This indicates that the administration of PRDNS had beneficial effects on some aspect of their rhinitis that bothered them, such as sleep, daily activities/sports, work, and troublesome symptoms.

Among the symptoms of AR, nasal blockage is a common problem, substantially impacting the quality of life of the patients $[19,20]$. All our patients complained of persistent NO. Therefore, in the present study, we used NIPF as an objective evaluation of NO. NIPF has been found to be an optimal variable to assess airflow for comparison over time, between subjects and in clinical trials [21]. As for NIPF, significant improvement $(p<0.001)$ was noted after 4 weeks of PRDNS administration, indicating a positive and objective effect on NO (Table 1; Fig. 2). No correlation between ARCT and NIPF was observed either before or after 30 days of PRDNS administration (data not shown). This is in line with previous studies looking at the correlation between the sensation of $\mathrm{NO}$ and the objective measure of nasal flow $[22,23]$.

Interestingly, the improvement in ARCT and NIPF observed after 30 days of PRDNS administration occurred in patients who remained uncontrolled despite PAR treatments over at least 1 year, with antihistamine alone or in combination with intranasal steroids - and for some of them, decongestants as needed. The significant improvement of these 2 outcomes following PRDNS administration may be related to the EOs contained in the nasal spray. Indeed, several components of EOs have demonstrated anti-inflammatory and anti-allergic effects. For example, 1.8 cineole - which is abundant in ravintsara, eucalyptus radiata, and niaouli, 3 EOs contained in PRDNS - decreases the production of inflammatory cytokines such as IL- $1 \beta$, TNF- $\alpha$, IL- 4 , and IL- 5 , which play an important role in the inflammatory allergic response [24, 25]. The effectiveness of inhalation of 1.8-cineol-rich EOs on NO in patients with PAR has also been recently demonstrated in a randomized placebo controlled trial [17].

In addition to the $4 \mathrm{EOs}$ (ravintsara, geranium, eucalyptus radiata, niaouli), hypertonic saline (13\%) may also have played a role in the improvement of ARCT and NIPF observed at D30. Indeed, saline irrigation with isotonic or hypertonic solutions is commonly used in vari-

Allergic Rhinitis and Essential Oils ous types of rhinitis, allergic or not, particularly for relief of NO. However, a recent systematic review of published randomized control trials [26] concluded that it was uncertain that saline, in addition to pharmacological treatment (antihistamines or intranasal steroids), improved symptoms or quality of life after 4 weeks to 3 months of administration compared with pharmacological treatments alone. As a large number of patients included in the present study had persistent rhinitis symptoms, particularly NO, while receiving antihistamine and nasal steroids, the role of saline alone in the improvement of ARCT and NIPF at D30 seems unlikely. Possible limitations of the study include the small sample size, the absence of a placebo control group (with hypertonic saline alone), and the short time of treatment and follow-up.

According to our hypothesis based on $20 \%$ improvement of ARCT, the primary outcome after 30 days of PRDNS administration, 60 patients were required. Due to enrollment issues, we decided to stop enrollment after 43 patients had been included. Despite this lower number of patients included, our data showed a marked significant improvement in ARCT and PRDNS. This suggests that the effect of PRDNS on these 2 outcomes, assumed to be $20 \%$ improvement from D0 to D30 to calculate the number of patients to treat, may have been underestimated. It is well known that the placebo effect has a potential influence on AR studies [27]. However, our aim was to evaluate the effect of the PRDNS spray longitudinally, and each patient was his own control. Furthermore, 40 patients among the 43 included were already treated for their AR and remained uncontrolled with bothersome NO. The latter was assessed using NIPF, which is an objective measurement of nasal patency [21]. Although we cannot exclude a possible placebo effect, the latter would not fully explain the improvement in NIPF observed after 30 days of PRDNS administration. Another possible limitation of the study is that seasonal changes might have contributed to symptoms improvement, especially in polysensitized patients. One last limitation is the short duration of treatment (30 days). We cannot assume that the benefit observed after 30 days would be maintained with a longer treatment. Nevertheless, the present work is a pilot study and further longer studies in patients with PAR are needed to assess the persistent benefit of the nasal spray.

Only 3 patients among the 43 had no treatment at inclusion in the study. Although these 3 patients exhibited a marked improvement in ARCT and NIPF after 30 days of PRDNS administration, a study in PAR naive patients would be necessary to assess the effectiveness of PRDNS compared to antihistamines and nasal corticosteroids. Fi-

Int Arch Allergy Immunol 2021;182:182-189 
nally, no moderate-to-severe side effects were noted, confirming the safety of PRDNS.

PAR is a significant health problem, which can significantly affect patient's quality of life. The current study showed the excellent tolerance of a nasal spray of hypertonic saline and EOs and found an association between its use and the reduction of symptoms in patients with PAR. Patient adherence and patient preferences related to medication selection may lead to better treatment outcomes. The PRDNS nasal spray is a natural and well-tolerated new option in the management of PAR. Further studies are needed to better determine its place in the PAR armamentarium.

\section{Statement of Ethics}

The study was approved by a local French Ethics Committee (Registration No. ID-RCB: 2018-A00836-49) and registered on ClinicalTrials.gov (No. NCT03963076). All participants signed an informed consent document, including consent for publication.

\section{Conflict of Interest Statement}

The authors have no conflicts of interest to declare. The funding source had no role in the design, conduct, or analysis of the study or the decision to submit the manuscript for publication.

\section{Funding Sources}

This study was supported by the company "Puressentiel."

\section{Author Contributions}

Conception and design: D.C., G.T., and P.D. Acquisition of data: D.C., C.N., R.L., and O.M. Analysis and interpretation of data: D.C., G.T., and P.D. Drafting or revising the manuscript for important intellectual content: D.C., C.N., R.L., O.M., G.T., and P.D. Final approval of the version to be published: D.C., C.N., R.L., O.M., G.T., and P.D. Agreement to be accountable for all aspects of the work: D.C., C.N., R.L., O.M., G.T., and P.D.

\section{References}

1 Small P, Keith PK, Kim H. Allergic rhinitis. Allergy Asthma Clin Immunol. 2018 Sep; 14(Suppl 2):51.

2 Dykewicz MS, Hamilos DL. Rhinitis and sinusitis. J Allergy Clin Immunol. 2010 Feb; 125(2 Suppl 2):S103-15.

3 Bousquet J, Neukirch F, Bousquet PJ, Gehano P, Klossek JM, Le Gal M, et al. Severity and impairment of allergic rhinitis in patients consulting in primary care. J Allergy Clin Immunol. 2006 Jan;117(1):158-62.

4 Bousquet J, Khaltaev N, Cruz AA, Denburg J, Fokkens WJ, Togias A, et al. Allergic rhinitis and its impact on asthma (ARIA)2008 update (in collaboration with the world health Organization, GA(2)LEN and AllerGen). Allergy. 2008 Apr;63(Suppl 86):8-160.

5 Demoly P, Allaert FA, Lecasble M. ERASM, a pharmacoepidemiologic survey on management of intermittent allergic rhinitis in every day general medical practice in France. Allergy. 2002 Jun;57(6):546-54.

6 Benninger M, Farrar JR, Blaiss M, Chipps B, Ferguson B, Krouse J, et al. Evaluating approved medications to treat allergic rhinitis in the United States: an evidence-based review of efficacy for nasal symptoms by class. Ann Allergy Asthma Immunol. 2010 Jan;104(1): 13-29.

7 Yanez A, Rodrigo GI. Intranasal corticosteroides versus topical $\mathrm{H} 1$ receptor antagonist for the treatment of allergic rhinitis: a systematic review with meta-analysis. Ann Allergy Asthma Immunol. 2002 Nov;89(5):479-84.
8 Pullerits T, Praks L, Ristioja V, Lötvall J. Comparison of a nasal glucocorticoid, antileukotriene, and a combination of antileukotriene and antihistamine in the treatment of seasonal allergic rhinitis. J Allergy Clin Immunol. 2002 Jun;109(6):949-55.

9 May JR, Dolen WK. Evaluation of intranasal corticosteroid sensory attributes and patient preference for fluticasone furoate for the treatment of allergic rhinitis. Clin Ther. 2019 Aug;41(8):1589-96.

10 Weiner JM, Abramson MJ, Puy RM. Intranasal corticosteroids versus oral $\mathrm{H} 1$ receptor antagonists in allergic rhinitis: systematic review of randomised controlled trials. BMJ. 1998 Dec;317(7173):1624-9.

11 El W, Harris WC, Babcock CM, Alexander $\mathrm{BH}$, Riley CA, McCoul ED. Epistaxis risk associated with intranasal corticosteroids sprays: a systemic review and meta-analysis. Otolaryngol Head Neck Surg. 2019 Jul;161(1): 18-27.

12 Qiu J, Grine K. Complementary and alternative treatment for allergic conditions. Prim Care. 2016 Sep;43(3):519-26.

13 Kem J, Bielory L. Complementary and alternative therapy (CAM) in the treatment of allergic rhinitis. Curr Allergy Asthma Rep. 2014 Dec;14(12):479.

14 Surda P, Fokkens WJ. Novel, alternative, and controversial therapies of rhinitis. Immunol Allergy Clin North Am. 2016 May;36(2):40123.
15 De Cassia da Silveira e Sà R, Andrade LN, de Sousa DP. A review on anti-inflammatory activity of monoterpenes. Molecules. 2013 Jan; 18(1):1227-54.

16 Ueno-Lio T, Shibakura M, Yokota K, Aoe M Hyoda T, Shinoata R, et al. Lavender essential oil inhalation suppresses allergic inflammation and mucus cell hyperplasia in a murine model of asthma. Life Sci. 2014 Jul;108(2): 109-15.

17 Choi Y, Park K. Effect of inhalation of aromatherapy oil with perennial allergic rhinitis: a randomized controlled trial. Evid Based Complement Alternat Med. 2016 Mar;2016: 2103616.

18 Demoly P, Jankowski R, Chassany O, Bessah $\mathrm{Y}$, Allaert FA. Validation of a self-questionnaire for assessing the control of allergic rhinitis. Clin Exp Allergy. 2011 Jun;41(6):8608.

19 Ottaviano G, Fokkens WJ. Measurements of nasal airflow and patency: a critical review with emphasis on the use of peak nasal inspiratory flow in daily practice. Allergy. 2016 Feb;71(2):162-74.

20 Ryden O, Andersson B, Andersson M. Disease perception and social behavior in persistent rhinitis: a comparison between patients allergic and nonallergic rhinitis. Allergy. 2004 Apr;59(4):461-4.

21 Braniuk JN. Subjective nasal fullness and objective congestion. Proc Am Thorac Soc. 2011 Mar;8(1):62-9. 
22 Panagou P, Loukides S, Tsipra S, Syrigou K, Anastasakis C, Kalogeropoulos N. Evaluation of nasal patency: comparison of patients and clinician assessments with rhinomanometry. Acta Otolaryngol. 1998 Nov;118(6):847-51.

23 Morrissey MS, Alun-Jones T, Hill J. The relationship of peak inspiratory airflow to subjective airflow in the nose. Clin Otolaryngol Allied Sci. 1990 Oct;15(5):447-51.
24 Juergens UR, Dethlefsen U, Steinkamp G, Gillissen R, Repges R, Vetter H. Anti-inflammatory activity of 1.8 cineol in bronchial asthma: a double-blind placebo controlled trial. Respir Med. 2003 Mar;97(3):250-6.

25 Bastos PD, Gomes AS, Lima FJB, Brito TS, Soares PM, Pinho JP, et al. Inhaled 1.8 cineole reduces inflammatory parameters in airways of ovalbumin-challenged guinea pigs. Basic Clin Pharmacol Toxicol. 2011 Jan;108(1):349.
26 Head K, Snidvongs K, Glew S, Scadding G, Schilder AG, Philpott C, et al. Saline irrigation for allergic rhinitis. Cochrane Database Syst Rev. 2018 Jun;6(6):CD012597.

27 Meltzer EO, Weiler JM, Widlitz MD. Comparative outdoor study of the efficacy, onset and duration of action, and safety of cetirizine, loratadine, and placebo for seasonal allergic rhinitis. J Allergy Clin Immunol. 1996 Feb;97(2):617-26. 